\title{
TEN WEEKS OF RAPID VENTRICULAR PACING CREATES A LONG-TERM MODEL OF LEFT VENTRICULAR DYSFUNCTION
}

\author{
Himanshu J. Patel, MDa \\ James J. Pilla, PhD $^{\mathrm{a}}$ \\ David J. Polidori, MDa \\ Sorin V. Pusca, MD \\ Theodore A. Plappert, CVT ${ }^{\mathrm{b}}$ \\ Martin St John Sutton, FRCP \\ Edward B. Lankford, $\mathrm{MD}, \mathrm{PhD}^{\mathrm{b}}$ \\ Michael A. Acker, MD
}

\begin{abstract}
Objective: Rapid ventricular pacing produces a reliable model of heart failure. Cessation after 4 weeks of rapid ventricular pacing results in rapid normalization of left ventricular function, but the left ventricle remains persistently dilated. We present novel data that show that prolonged rapid ventricular pacing (10 weeks) creates a model of chronic left ventricular dysfunction.
\end{abstract}

Methods: In 9 dogs undergoing 10 weeks of rapid ventricular pacing, left ventricular function and volumes were serially assessed by using 2-dimensional echocardiography and pressure-volume analysis for 12 weeks after cessation of pacing.

Results: Increased end-diastolic volume and decreased systolic and diastolic function were seen at the end of pacing. By 2 weeks of recovery from rapid ventricular pacing, end-diastolic volume and ejection fraction were partially recovered but did not improve further thereafter. Load-independent and loadsensitive indices of function obtained by pressure-volume analysis at 8 and 12 weeks of recovery confirmed a persistence of both systolic and diastolic dysfunction. In addition, left ventricular mass increased with pacing and remained elevated at 8 and 12 weeks of recovery. Four of these dogs studied at 6 months of recovery showed similar left ventricular abnormalities.

Conclusion: Ten weeks of rapid ventricular pacing creates a long-term model of left ventricular dysfunction. (J Thorac Cardiovasc Surg 2000;119:834-41)
$\mathrm{T}^{\mathrm{s}}$ study new surgical therapies for end-stage dilated cardiomyopathy, a stable, long-term animal model analogous to the human condition needs to be developed. One potential model is rapid ventricular pacing. This model produces a reliable model of left ventricular (LV) dysfunction and leads to heart failure (HF) within 1 to 2 weeks of initiating pacing. ${ }^{1-6}$ Congestive $\mathrm{HF}$ develops with signs of venous congestion, systolic and diastolic LV dysfunction, and an increase in ventricular volumes. $^{1-8}$ Neurohumoral profiles obtained in this model have shown abnormalities consistent with an activated sympathetic system. ${ }^{2,3,9}$ Most reports have

From the Divisions of Cardiothoracic Surgery a and Cardiology, University of Pennsylvania School of Medicine, Philadelphia, Pa.

Received for publication Nov 17, 1998; revisions requested Feb 19, 1999; revisions received Oct 20, 1999; accepted for publication Nov 23, 1999.

Address for reprints: Michael A. Acker, MD, Associate Professor of Surgery, Division of Cardiothoracic Surgery, Hospital of the University of Pennsylvania, Silverstein 4, 3400 Spruce St, Philadelphia, PA 19104.

Copyright $\odot 2000$ by The American Association for Thoracic Surgery. $0022-5223 / 2000 \$ 12.00+0 \quad \mathbf{1 2 / 1 / 1 0 4 5 8 0}$

doi: $10.1067 / \mathrm{mtc} .2000 .104580$ used pacing protocols for 2 to 4 weeks. Several recent studies have assessed the short-term recovery (typically 1 month) after cessation of pacing. 1,3-5,8-15 Unfortunately, they have shown that termination of rapid ventricular pacing results in a rapid normalization of load-sensitive indices of global cardiac function, neurohumoral profiles, and hemodynamics, whereas abnormalities of cardiac size and individual myocyte function persist.

We hypothesized that prolonging the insult to the myocardium would result in more significant changes in the cellular and molecular processes inducing HF in this model, and more severe permanent LV dysfunction would then result. Accordingly, this study was conducted to assess the long-term recovery after prolonged rapid ventricular pacing. This study used serial echocardiography and pressure-volume (PV) analysis to obtain novel longitudinal information about cardiac function, volumes, and energetics after cessation of prolonged rapid ventricular pacing.

\section{Material and methods}

All animals used in this study received care in compliance with the "Guide for the Care and Use of Laboratory Animals" 
prepared by the Institute of Laboratory Animal Resources, National Research Council, and published by the National Academy Press, revised 1996, and the investigation was approved by the Institutional Animal Care and Use Committee of the University of Pennsylvania.

Experimental design. Nine heartworm-free adult mongrel dogs (22-30 kg) were studied. All dogs underwent baseline 2dimensional echocardiography and PV analysis as described below. After pacemaker implantation, the dogs were subjected to rapid ventricular pacing at 215 beats/min for 10 weeks to ensure development of HF. At the end of pacing, studies were repeated in all dogs. Pacemakers were removed at peak failure (HF). Seven of 9 dogs ( 2 dogs were killed at the 10week study point of rapid ventricular pacing because of complications during data collection) were observed during recovery from rapid ventricular pacing by using biweekly echocardiograms for 8 weeks, and a final echocardiogram was obtained at 12 weeks. PV analysis was conducted in these 7 dogs at 8 and 12 weeks of recovery. Two additional dogs underwent echocardiograms and PV analysis only at 12 weeks of recovery. Terminal studies in a subset of these dogs $(\mathrm{n}=4)$ were carried out at 6 months after cessation of rapid ventricular pacing. Symptoms of cardiac failure (extremity edema, ascites, and anorexia) present in all dogs by the time of peak failure were treated with daily digoxin $(0.125 \mathrm{mg})$ and furosemide $(20 \mathrm{mg})$ along with thrice weekly aspirin (80 $\mathrm{mg}$ ) for the last 9 weeks of the rapid pacing period and the first week of recovery.

Echocardiography. Each animal was mildly sedated with acepromazine $(0.05 \mathrm{mg} / \mathrm{kg})$ before we obtained echocardiograms. Two-dimensional transthoracic echocardiograms taken with an ACUSON 128XP echocardiograph (Acuson Corporation, Mountain View, Calif) were then obtained under awake conditions. Both short-axis and apical 4-chamber and long-axis views were obtained and stored for later analysis.

Anesthesia. One hour before either PV analyses or pacemaker insertions, each animal was premedicated intramuscularly with acepromazine $(0.1 \mathrm{mg} / \mathrm{kg})$ and glycopyrrolate (INN: glycopyrronium bromide; $0.001 \mathrm{mg} / \mathrm{kg}$ ). General anesthesia was induced with ketamine $(10 \mathrm{mg} / \mathrm{kg})$ and diazepam $(0.5 \mathrm{mg} / \mathrm{kg})$ administered intravenously and maintained after endotracheal intubation with inhaled oxygen $(3 \mathrm{~L} / \mathrm{min})$ and isoflurane (1\%-2\%). Cefazolin $(25 \mathrm{mg} / \mathrm{kg}$ administered intravenously) was given prophylactically before the skin incision and again just before the termination of all procedures.

PV analysis. PV analysis was conducted after achievement of anesthesia as follows. A 7F multielectrode dual-field conductance catheter (Sentron Europe BV, Maastricht, The Netherlands) and a $5 \mathrm{~F}$ micromanometer-tipped catheter (Millar Instruments, Inc, Houston, Tex) were placed under fluoroscopic guidance along the long axis of the LV cavity by sterile cutdowns of the carotid and right femoral arteries.

Similarly, 20-mL occlusion catheters (Applied Vascular, Laguna Hills, Calif) were placed at the junction of the superior and inferior venae cavae with the right atrium through the right jugular and right femoral veins. A balloon-tipped pulmonary artery catheter was placed through the left jugular vein.
Volume measurements were obtained by using the conductance catheter technique. ${ }^{16-22}$ All hemodynamic signals and the electrocardiographic tracing were processed by using an analog amplifier (Gould, Inc, Cleveland, Ohio) digitized at $200 \mathrm{~Hz}$ and stored on computer disk for offline analysis.

All data were collected with the ventilator held at end-expiration. For determination of the end-systolic and end-diastolic PV relationships and the preload recruitable stroke work (PRSW) relationship ${ }^{23}$ (the slope of stroke work vs enddiastolic volume [EDV]), the $20-\mathrm{mL}$ balloons in the venae cavae were temporarily inflated to alter preload, as described previously. All incisions were then closed primarily.

Pacemaker insertion. After a recovery period of no less than 3 days after the initial PV analysis, each dog was administered general anesthesia and underwent placement of specially modified ventricular pacemakers (model IS-1; Medtronics, Inc, Minneapolis, Minn) designed to maintain prolonged pacing rates at 215 beats $/ \mathrm{min}$. Through a right anterior thoracotomy, a $2-\mathrm{cm} \times 3-\mathrm{cm}$ section of the apical pericardium was excised, and a unipolar pacing lead (model IS-1, Medtronics, Inc) was secured to the LV apex. The lead was then tunneled subcutaneously to a subfascial pocket under the right rectus abdominis and connected to the implantable pacemaker. A right thoracostomy tube was then placed, and all incisions were closed in layers. The tube was removed after 1 hour while the dog was under anesthesia. Animals were allowed to recover for at least 7 days, after which pacing was initiated at a rate of 215 beats/min for 10 weeks.

Data analysis. Two-dimensional echocardiographic LV volumes were calculated by using the modified biplane Simpson rule. ${ }^{24}$ Cardiac shape was assessed by examining the ratio of the short-to-long axis area in diastole. All hemodynamic data were analyzed offline by using custom software. Parallel conductance from extracavitary structures was derived from saline calibration, as previously described. ${ }^{19}$ Volume gain was set to unity. Measured parameters were heart rate, stroke volume, peak and end-diastolic LV pressures, and pulmonary artery pressures. Derived parameters included cardiac output, stroke work, maximum and minimum rates of pressure change (maximum $\mathrm{dP} / \mathrm{dt}$ and minimum $\mathrm{dP} / \mathrm{dt}$ ), and the time constant of isovolumic pressure decay $(\tau)$. This constant was calculated by using a nonlinear regression, as previously described. ${ }^{25}$ The end-systolic and end-diastolic PV relationships and the PRSW data were derived by using the caval inflow occlusion data. ${ }^{19}$ These curves were obtained from data taken after the initial decline seen in the LV pressure. All data in which there was a change in the heart rate of over 5\% was discarded to minimize the effects of cardiovascular reflexes; indices of myocardial energetics, including PV area (correlating with oxygen consumption); external mechanical work (area circumscribed by the PV loop); and the potential energy (dissipated as heat during contraction) were obtained from representative end-systolic and end-diastolic relationships as described by Suga. ${ }^{26}$ Myocardial mechanical efficiency was defined as the ratio of the external work to the total PV area. 


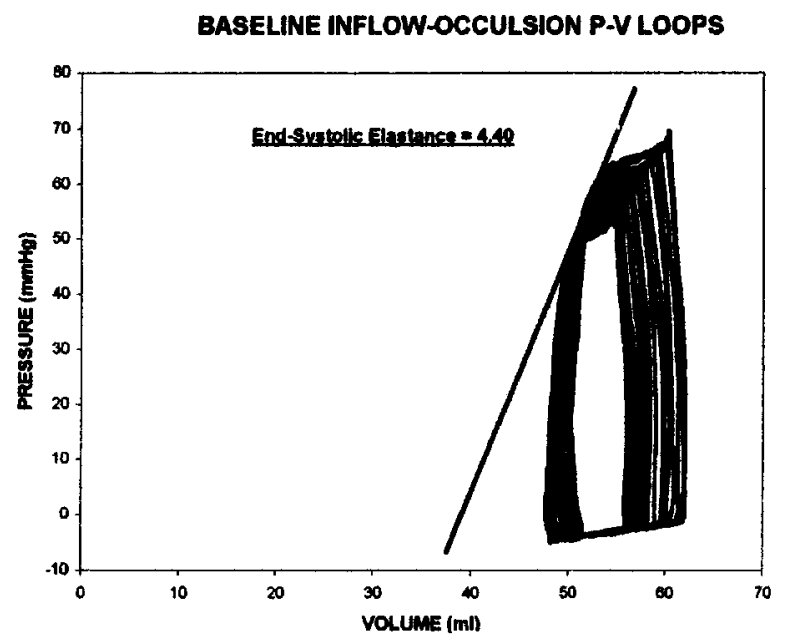

10 WEEKS RVP INFLOW-OCCLUSION P.V LOOPS

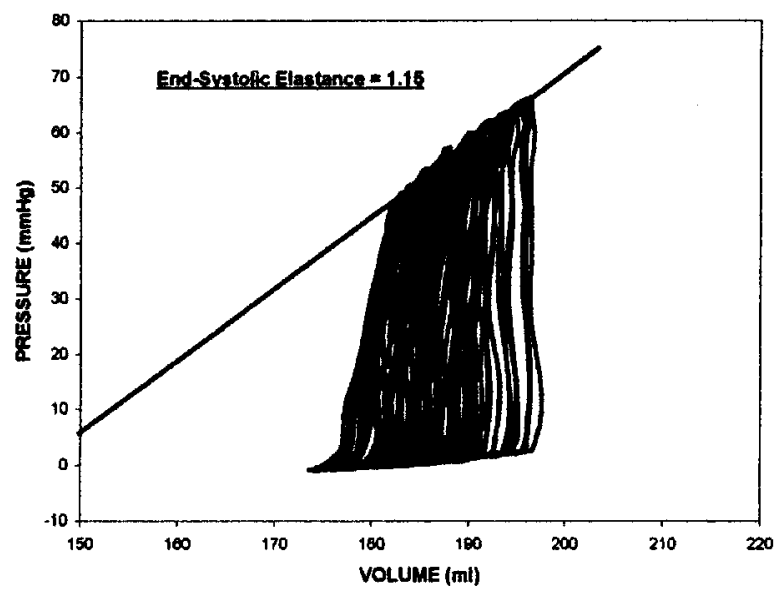

3 MONTHS POST-RVP INFLOW-OCCLUSION P-V LOOPS

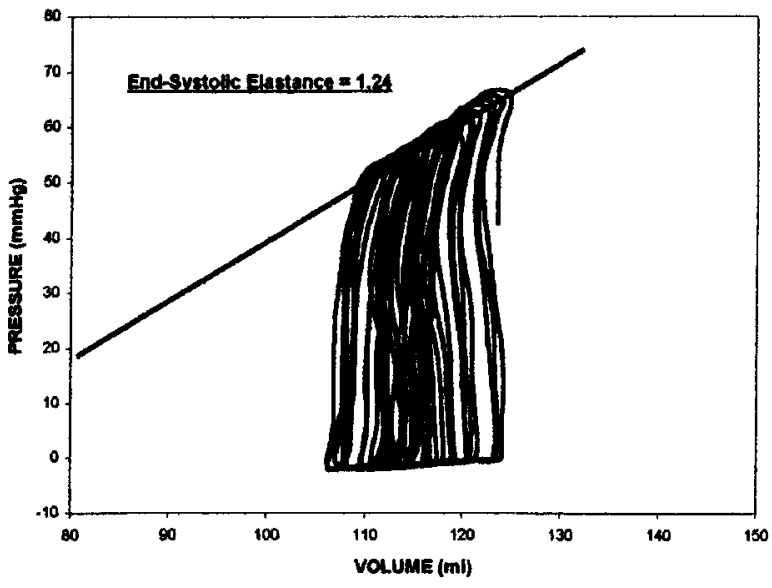

Fig 1. End-systolic elastance pressure-volume $(P V)$ loops. Inflow occlusion PV loops obtained from one dog. The slope of the end-sysolic PV relationship decreases at peak HF compared with baseline. The decreased slope persists at 3 months of recovery.

Statistical tests were performed by using standard computer software (SigmaStat; Jandel Scientific, San Rafael, Calif; and STATISTICA; StatSoft Inc, Tulsa, Okla). All data are expressed as means \pm SEM. End-systolic elastance and PRSW were assessed by using analysis of covariance with changing covariates (volume axis intercept [Vo] and EDV axis intercept $[\mathrm{Mo}]$ ) and repeated measures followed by the Newman-Keuls multiple comparison method. All other differences between time points were assessed by using analysis of variance with repeated measures followed by the multiple comparison method of Newman and Keuls.

\section{Results}

PV loops obtained from one dog are shown in Fig 1. These loops are representative of an inflow occlusion study that was used to calculate end-systolic elastance. The slope of the end-systolic PV relationship decreased with 10 weeks of pacing and remained depressed at 3 months of recovery.

Creation of HF. By the end of pacing (HF), LV function had deteriorated significantly from baseline (Figs 2, 3, and 4). Signs indicative of cardiac failure, including ascites, anorexia, inspiratory crackles, and a third heart sound, were present in all dogs by the end of pacing. Systolic parameters of PRSW, end-systolic elastance, maximum $\mathrm{dP} / \mathrm{dt}$, and echocardiographic ejection fraction were all depressed (PRSW, $P=.000003$; endsystolic elastance, $P=.000619$; maximum $\mathrm{dP} / \mathrm{dt}, P=$ .0000008 ; ejection fraction, $P=1.45 \times 10^{-14}$; Fig 3). Similarly, diastolic dysfunction was evident by a sig- 
nificant rise in relaxation rates and minimum $\mathrm{dP} / \mathrm{dt}(\tau$, $P=.0013$; minimum dP/dt, $P=.00005 ;$ Fig 4 ). LV enddiastolic pressure had increased by $155 \%(P<.05$ vs baseline, Fig 4). Chamber EDV had increased significantly from $69.5 \pm 4.1 \mathrm{~mL}$ to $125.3 \pm 5.5 \mathrm{~mL}(P<.05$, Fig 2). LV mass increased significantly and remained elevated throughout the recovery period $(P=.0000007$, Fig 5). Cardiac output measured by thermodilution decreased from $3603 \pm 562 \mathrm{~mL}$ at baseline to $1908 \pm$ $167 \mathrm{~mL}$ at peak HF $(P<.05)$. Conversely, cardiac output returned to baseline levels at 2 months of recovery.

Echocardiographic time course of recovery. Cessation of pacing led to an increase in LV systolic performance with small changes in LV chamber size (Fig 2). At 2 weeks of recovery, EDVs decreased to $92 \%$ of peak failure volumes $\left(P=7 \times 10^{-15}\right)$, whereas ejection fraction increased to $40.1 \% \pm 2.2 \%$ ( $P<.05 \mathrm{vs}$ $\mathrm{HF}$ and baseline). However, as Fig 2 shows, reverse remodeling and improvements in ejection fraction were halted by 2 weeks of recovery. EDV and ejection fraction at each subsequent biweekly interval continued to be significantly different from baseline $(P<.05)$.

PV analysis of recovery. $\mathrm{PV}$ analysis conducted at 8 weeks of recovery showed that the load-independent index of contractility (PRSW) had also improved from peak HF $(P<.05$, Fig 3$)$ but still remained depressed compared with baseline values $(P<.05)$. Maximum $\mathrm{dP} / \mathrm{dt}$ and end-systolic elastance at 8 weeks were similar to those at peak failure $(P=$ not significant) and were significantly different from baseline values $(P<$ .05). Similarly, diastolic dysfunction was indicated by a persistently elevated relaxation rate and minimum $\mathrm{dP} / \mathrm{dt}(P<.05$ vs HF, and $P<.05$ vs baseline; Fig 4$)$. LV end-diastolic pressure had, however, returned to baseline levels.

PV analysis at 12 weeks of recovery revealed no significant change in systolic indices of function (end-systolic elastance, PRSW, and maximum $\mathrm{dP} / \mathrm{dt}$ : all $P=$ not significant vs 8 weeks of recovery). In addition, all systolic indices were different from baseline values $(P<$ $.05)$. Diastolic dysfunction was also persistent $(P<.05$ vs baseline). Finally, in a subset of dogs $(n=4)$ studied at 6 months after cessation of pacing, systolic and diastolic indices were similar to those seen at 12 weeks of recovery. The nadir values of all indices at peak failure for these 4 dogs were within the range seen for the group.

Effects of recovery on myocardial energetics. At peak HF, there was a 37\% decrease in stroke work compared with baseline (external work, $P=.05$; Table I). In addition, the potential energy that was generated and presumably dissipated as heat increased significantly

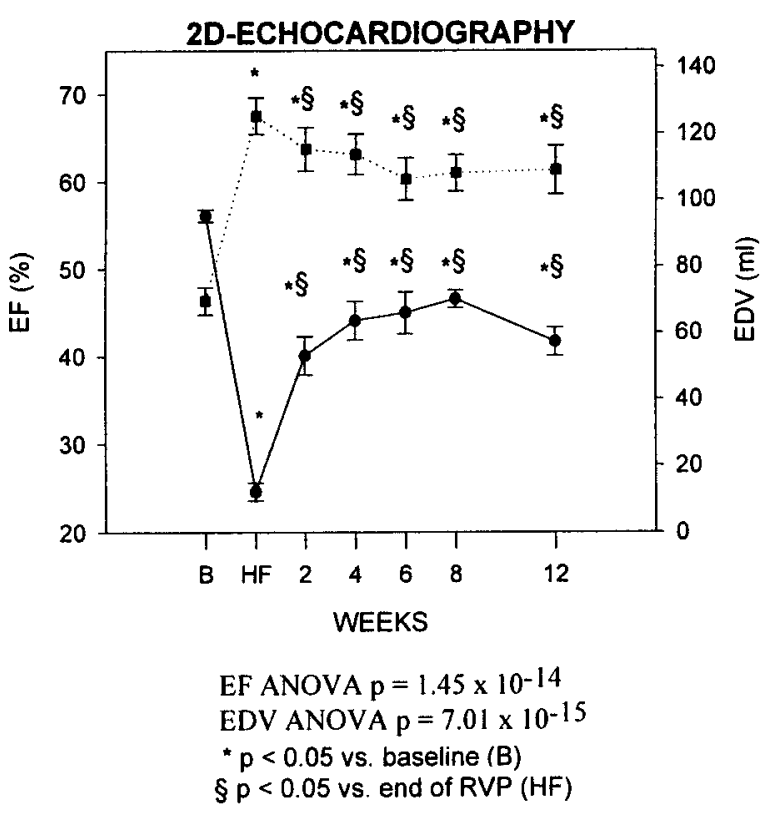

Fig 2. Echocardiographic time course of recovery after cessation of rapid ventricular pacing. Biweekly echocardiograms assessed recovery from pacing-induced failure in 7 dogs. By peak failure $(H F)$, there is a significant decrease in ejection fraction $(E F)$ and an increase in end-diastolic volume $(E D V)$. By 2 weeks, EF partially improves $(P<.05$ vs HF and baseline $[B])$, but there is no further improvement thereafter $(P=$ not significant for 2 weeks vs 4 weeks, 4 weeks vs 6 weeks, 6 weeks vs 8 weeks, and 8 weeks vs 12 weeks). A similar pattern is seen for EDV, where partial recovery is apparent by 2 weeks, but EDV is stable thereafter. $2 D$, Two-dimensional; ANOVA, analysis of variance.

by $86 \%$ (potential energy, $P=.01$ ). These opposite changes in the determinants of total PV area (a correlate of oxygen consumption) caused it to increase by $24 \%$, but this was not significant. However, myocardial mechanical efficiency, defined as the external work (stroke work) done from the total energy within the system (total PV area), declined significantly $(P=$ $.00002)$.

After cessation of pacing, at both 8 and 12 weeks of recovery, stroke work marginally improved $(P=$ not significant vs HF for 8 weeks and $P<.05$ vs HF for 12 weeks; Table I), but potential energy remained stable. As a result, total PV area and myocardial efficiency both marginally increased in parallel $(P=$ not significant vs HF for 8 or 12 weeks of recovery).

\section{Discussion}

Chronic tachycardia-induced HF is a reliable and reproducible model of biventricular dilated cardiomy- 

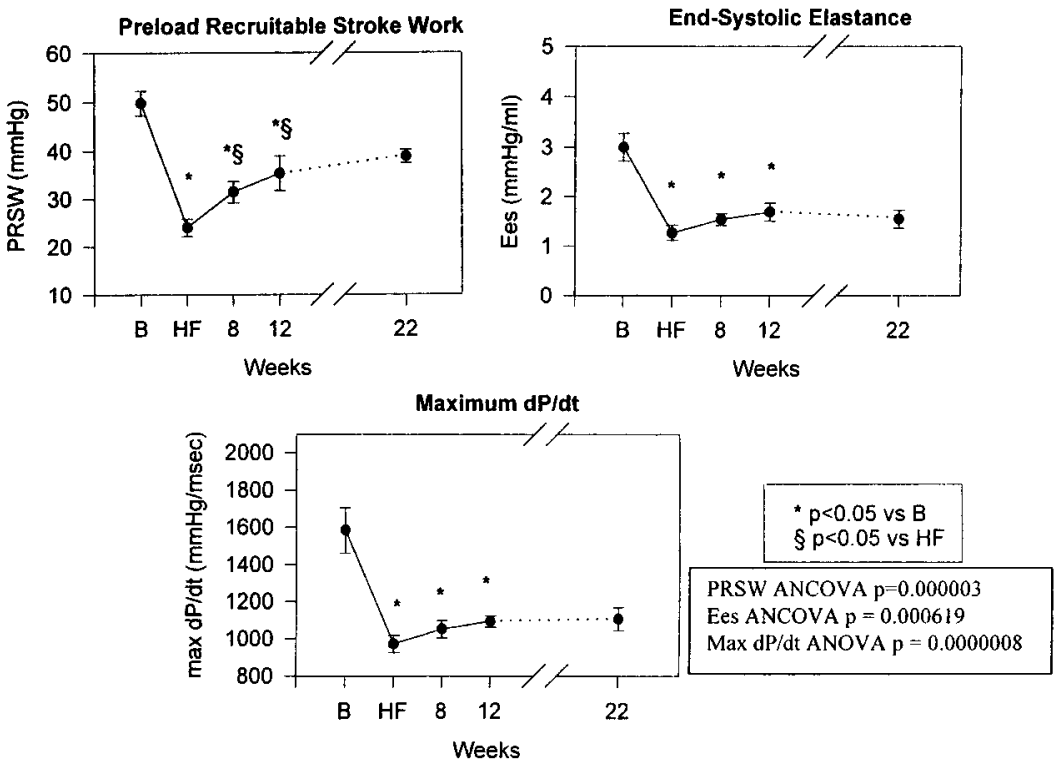

Fig 3. Recovery of indices of contractility after cessation of pacing. In 9 dogs undergoing PV analysis, contractility indices of end-systolic elastance (Ees) and maximum $\mathrm{dP} / \mathrm{dt}$ do not significantly improve after termination of pacing. Preload recruitable stroke work $(P R S W)$, however, does, but at 8 and 12 weeks of recovery it is still significantly different from baseline values. There is no significant difference between 8 and 12 weeks of recovery in any of these indices of contractility. In a subset of these dogs $(n=4)$, PV analysis carried out at 22 weeks of recovery (dotted lines) showed no further improvement. Indices of LV systolic function at peak failure in this subset were within the range seen for the group as a whole. $B$, Baseline; $H F$, heart failure; ANCOVA, analysis of covariance; ANOVA, analysis of variance.
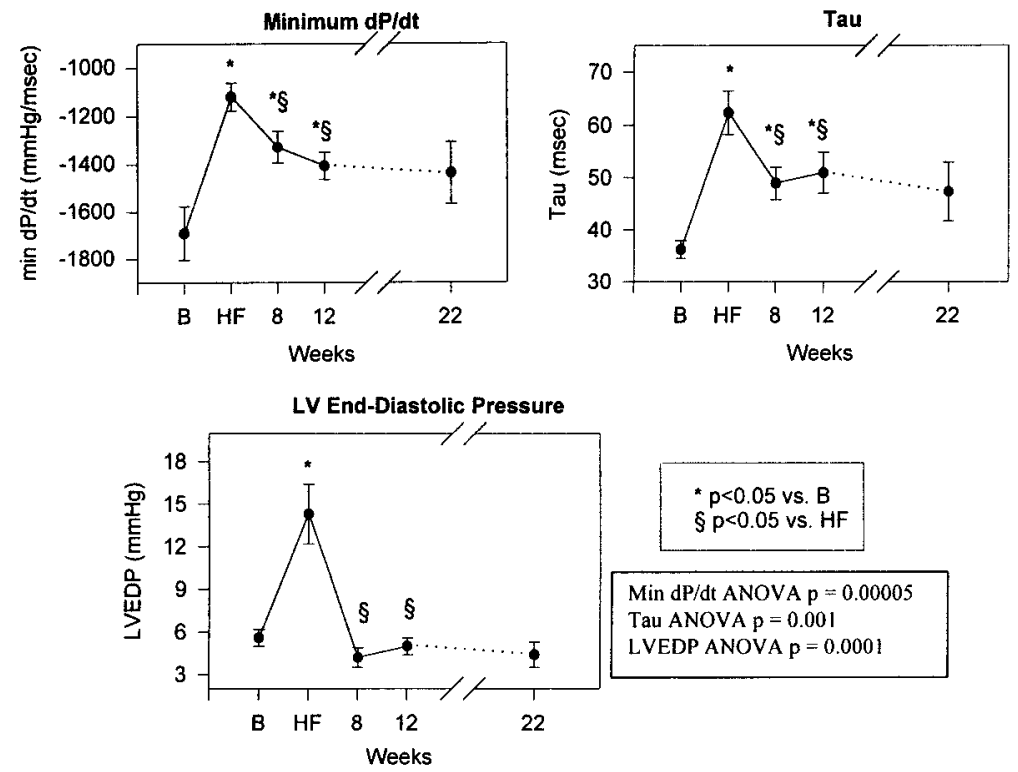

Fig 4. Diastolic indices after prolonged tachycardia. In 9 dogs undergoing PV analysis, diastolic indices of minimum $\mathrm{dP} / \mathrm{dt}$ and $\tau$ partially improve after termination of pacing but are still different from baseline $(B)$. In a subset of dogs $(n=4)$ studied at 22 weeks of recovery (dotted lines), no further improvement was seen. LV end-diastolic pressure (LVEDP), however, returns to baseline values at 8 weeks of recovery and remains normal in the subset of dogs studied at 22 weeks. Diastolic indices at peak failure in this subset were within the range seen for the whole group. ANOVA, Analysis of variance. 


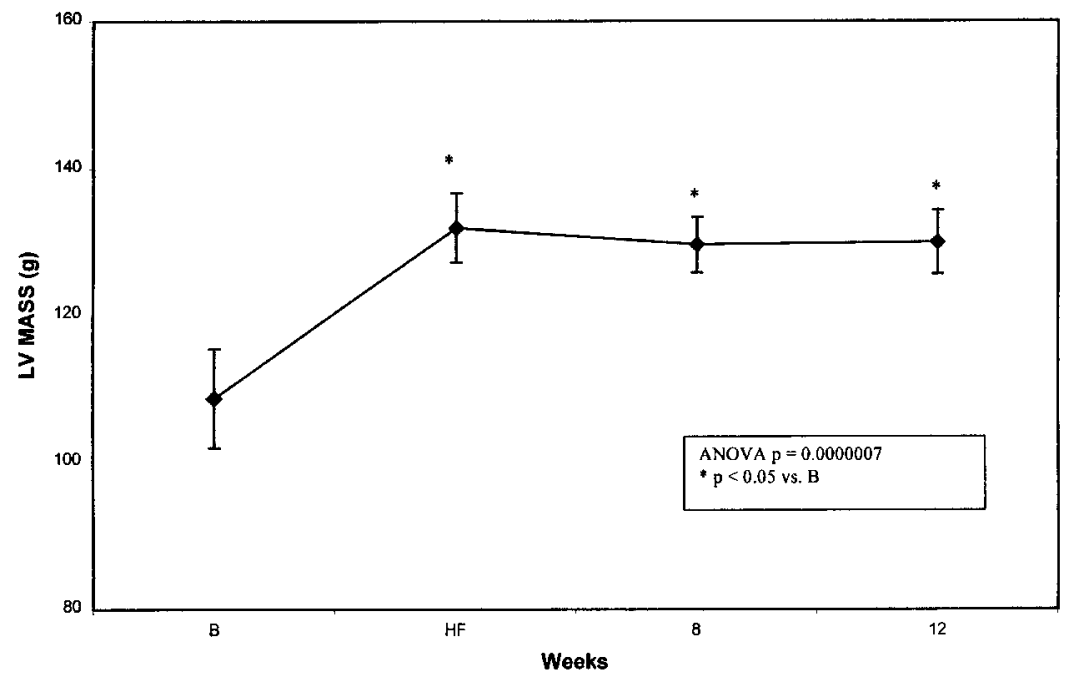

Fig 5. LV hypertrophy. The LV mass, measured by means of echocardiography, increased significantly at peak heart failure when compared with baseline values $(B ; P=.0000007)$. The hypertrophy continued at 8 and 12 weeks of recovery.

Table I. Indices of myocardial energetics

\begin{tabular}{|c|c|c|c|c|c|}
\hline Parameter & Baseline & Peak failure & 8-wk recovery & 12-wk recovery & $\begin{array}{l}\text { P values } \\
\text { (ANOVA) }\end{array}$ \\
\hline $\mathrm{EW}(\mathrm{mm} \mathrm{Hg} \cdot \mathrm{mL})$ & $1266 \pm 173$ & $796 \pm 120^{*}$ & $970 \pm 111$ & $1184 \pm 136^{\dagger}$ & .05 \\
\hline $\mathrm{PE}(\mathrm{mm} \mathrm{Hg} \cdot \mathrm{mL})$ & $1146 \pm 139$ & $2139 \pm 200^{*}$ & $2054 \pm 256^{*}$ & $2245 \pm 355^{*}$ & .015 \\
\hline $\mathrm{PV}$ area $(\mathrm{mm} \mathrm{Hg} \cdot \mathrm{mL})$ & $2365 \pm 241$ & $2935 \pm 286$ & $3023 \pm 338$ & $3372 \pm 413$ & .2 \\
\hline Efficiency $(\%)$ & $53.1 \pm 5.0$ & $26.8 \pm 2.1^{*}$ & $32.2 \pm 2.0^{*}$ & $35.9 \pm 3.2^{*}$ & .00002 \\
\hline
\end{tabular}

All data are expressed as mean \pm SEM. ANOVA, Analysis of variance; $E W$, external work; $P E$, potential energy; PV, pressure-volume.

${ }^{*} P<.05$ versus baseline, as determined by repeated-measures analysis of variance followed by the Newman-Keuls multiple comparison procedure.

$\dagger P<.05$ versus peak failure, as determined by repeated-measures analysis of variance followed by the Newman-Keuls multiple comparison procedure.

opathy. Within 1 week of pacing, systolic and diastolic dysfunction are evident, and sequelae of circulatory overload are seen shortly thereafter. ${ }^{1-5}$ Cardiac volumes rise progressively throughout the pacing period. ${ }^{1,4,5,7,11,13}$ Loss of compensation in failure is characterized by elevation of circulating neurohormones, including catecholamines, atrial natriuretic peptide, renin, angiotensin II, and aldosterone. ${ }^{3-6}$ On a cellular and molecular level, chronic tachycardia results in abnormalities in $\mathrm{Ca}^{++}$handling, leading to systolic and diastolic dysfunction ${ }^{27-31}$; disruption of the extracellular matrix, leading to myocyte slippage with concomitant increases in cardiac volumes ${ }^{4,32,33}$; and cytoskeletal aberrations, leading to systolic dysfunction. ${ }^{4}$

Cessation of 2 to 4 weeks of pacing, however, is characterized by a spontaneous diuresis that reduces filling pressures to normal levels within 72 hours. ${ }^{1,3,4,9}$ Systolic function also rapidly returns to normal within 1 week, ${ }^{8}$ although Spinale and coworkers ${ }^{13}$ have shown persistent myocyte shortening abnormalities at 4 weeks of recovery. Prior studies have also shown disparate recovery of systolic and diastolic function. ${ }^{13,15}$ In contrast to load-sensitive systolic indices, parameters of relaxation remain altered at up to 4 weeks of recovery. Diastolic volumes also remain elevated, and there is a compensatory hypertrophy after discontinuation of pacing that presumably results in a return of normal wall stresses. ${ }^{13,15}$

We hypothesized that prolonged rapid ventricular pacing may induce either more severe or more permanent changes in the myocardium when compared with standard shorter pacing protocols. Serial evaluation of the heart in this study extended long beyond the cessation of pacing. The data presented here describe novel findings that after prolonged tachycardia, global systolic and diastolic LV function remains different from baseline for an extended period of time. In contrast to other reports with shorter intervals of rapid ventricular 
pacing, we show that maximum positive and negative $\mathrm{dP} / \mathrm{dt}$ show a small rise. PV analysis showed that the end-systolic elastance and PRSW, which are relatively load-independent indices of contractility, were also abnormal. Longitudinal analysis revealed that there is only partial LV recovery after discontinuation of pacing seen in this study. The partial recovery that is seen is essentially complete within 2 weeks. Also, indices of myocardial energetics revealed a decrease in contraction efficiency at peak failure. This improved during recovery, although not significantly, and was mediated in part by an increase in stroke work, without a concomitant decrease in wasted potential energy (ie, heat dissipated during contraction).

Consistent with past reports, persistent LV diastolic dysfunction during recovery was demonstrated in this study. ${ }^{13-15}$ This persistent dysfunction may be due to permanent changes that occur in the extracellular matrix (altering EDV), as well as in intracellular $\mathrm{Ca}^{++}$ handling (affecting intrinsic myocyte relaxation). Although the commonly measured relaxation parameters are partly dependent on loading conditions, prolonged relaxation seen after cessation of pacing is likely due to abnormal intrinsic myocyte relaxation. ${ }^{4,13}$ In contrast, significant dysfunction in systole seen long after cessation of pacing in this study may require prolonged pacing. The additional insult of several extra weeks of pacing may be necessary to induce permanent changes in $\mathrm{Ca}^{++}$homeostasis and sarcolemmal function known to occur during rapid pacing. ${ }^{29,30}$

Potential concerns include that this study was artificially terminated at 12 weeks of recovery and that additional improvement in function could occur thereafter. However, in a subset of dogs studied by PV analysis 6 months after cessation of rapid ventricular pacing, systolic and diastolic dysfunction was confirmed. Although statistical comparisons were not possible in this subset, the values of indices of function were within the range seen at 12 weeks of recovery and were different from baseline. Thus we believe that the recovery after prolonged pacing is incomplete and that this is a long-term model of LV dysfunction.

Additional studies assessing cardiac responsiveness to $\beta$-adrenergic stimulation, as well as alterations in the neurohormonal status during recovery from prolonged pacing, would provide further insight into recovery from prolonged pacing. As has been shown after cessation of shorter pacing protocols, however, it is likely that $\beta$-receptor response dysfunction is also present in this model. ${ }^{13}$

In summary, we describe novel data on the long-term recovery after cessation of prolonged rapid pacing, showing persistence of significant systolic and diastolic dysfunction, as well as increased ventricular volumes. We also demonstrate that partial reverse remodeling with concomitant improvement in function in this model is complete by 2 weeks of recovery. The stability and the persistent dilation, as well as LV systolic and diastolic dysfunction, make it a suitable model for the study of novel surgical and mechanical therapies for end-stage dilated cardiomyopathies.

We thank Mr Randy Rossi, Dr Y-G Liu, and Mr James Wood for their excellent technical assistance. We also thank Medtronic, Inc, for providing part of the technical equipment needed for this study.

\section{REFERENCES}

1. Wilson JR, Douglas P, Hickey WF, Lanoce V, Ferrari N, Muhammed A, et al. Experimental congestive heart failure produced by rapid ventricular pacing in the dog: cardiac effects. Circulation 1987;75:857-67.

2. Armstrong PW, Stopps TP, Ford SE, de Bold AJ. Rapid ventricular pacing in the dog: patho-physiologic studies of heart failure. Circulation 1986;74:1075-84.

3. Riegger AJG, Liebau G. The renin-angiotensin-aldosterone system, antidiuretic hormone and sympathetic nerve activity in an experimental model of congestive heart failure in the dog. Clin Sci 1982;62:465-9.

4. Spinale FG. Pathophysiology of tachycardia induced heart failure. Mount Kisco (NY): Futura; 1996.

5. Shinbane JS, Wood MA, Jensen DN, Ellenbogen KA, Fitzpatrick AP, Scheinman MM. Tachycardia-induced cardiomyopathy: a review of animal models and clinical studies. J Am Coll Cardiol 1997;29:709-15

6. Travaill CM, Williams TDM, Pate P, Song G, Chalmers J, Lightman SL, et al. Haemodynamic and neuro humoral reponse in heart failure produced by rapid ventricular pacing. Cardiovasc Res 1992;26:783-90.

7. Scott BD, Sharma MK, Levett JM, Marinelli CC, Kieso RA, Schmid PG, et al. Cardiac geometry and mass changes associated with pacing-induced cardiomyopathy in the dog. Am Heart $\mathbf{J}$ 1993;125:1047-53.

8. Howard RI, Stopps TP, Moe GW, Gotlieb A, Armstrong PW. Recovery from heart failure: structural and functional analysis in a canine model. Can J Physiol Pharmacol 1989;66:1505-12.

9. Fitzpatrick MA, Nicholls MG, Espiner EA, Ikram H, Bagshaw P, Yandle RG. Neurohumoral changes during onset and offset of ovine heart failure: role of ANP. Am J Physiol 1989;256:H1052-9.

10. Moe GW, Stopps TP, Howard RJ, Armstrong PW. Early recovery from heart failure: insights into the pathogenesis of experimental chronic pacing induced heart failure. J Lab Clin Med 1988;112: 426-32.

11. Damiano RJ, Tripp HF, Asano T, Small KW, Jones RH, Lowe JE. Left ventricular dysfunction and dilation resulting from chronic supraventricular tachycardia. J Thorac Cardiovasc Surg 1987;94: $135-43$.

12. Larosa G, Armstrong PW, Seeman P, Forster C. Beta-adrenoreceptor recovery after heart failure in the dog. Cardiovasc Res 1993;27:489-93. 
13. Spinale FG, Holzgrefe HH, Mukherjee R, Arthur SR, Child MJ, Powell JR, et al. Left ventricular and myocyte structure and function after early recovery from tachycardia induced cardiomyopathy. Am J Physiol 1995;268:H836-47.

14. Moe GW, Grima EA, Howard RJ, Seth R, Armstrong PW. Left ventricular remodeling and disparate changes in contractility and relaxation during development of and recovery from experimental heart failure. Cardiovasc Res 1994;28:66-71.

15. Tomita M, Spinale FG, Crawford FA, Zile MR. Changes in left ventricular volume, mass, and function during the development and regression of supraventricular tachycardia-induced cardiomyopathy. Circulation 1991;83:635-44.

16. Baan J, Jong TTA, Kerkhof PLM, Moene RJ, van Dijk AD, van der Velde ET, et al. Continuous stroke volume and cardiac output from intra-ventricular dimensions obtained with the impedance catheter. Cardiovasc Res 1981;15:328-34.

17. Baan J, van der Velde ET, de Bruin HG, Smeek GJ, Koops J, van Dijk AD, et al. Continuous measurement of the left ventricular volume in animals and humans by the conductance catheter. Circulation 1984;70:812-23.

18. Steendjik P, van der Velde ET, Baan J. Left ventricular stroke volume by single and dual field excitation of conductance catheter in dogs. Am J Physiol 1993;264:H2198-207.

19. Kass DA. Clinical evaluation of left heart function by conductance catheter technique. Eur Heart J 1992;13(Suppl E): 57-64.

20. Burkhoff D. The conductance method of left ventricular volume estimation-methodologic limitations put into perspective. Circulation 1990;81:703-6.

21. Kass DA, Yamazaki T, Burkhoff D, Maughan WL, Sagawa K. Determination of left ventricular end-systolic pressure-volume relationships by the conductance (volume) catheter technique. Circulation 1986;73:586-95.

22. Sagawa K, Maughan L, Suga H, Sunagawa K. Cardiac contraction and the pressure-volume relationship. New York: Oxford University Press; 1988.
23. Glower DD, Spratt JA, Snow ND, Kabas JS, Davis JW, Olsen $\mathrm{CO}$, et al. Linearity of the Frank-Starling relationship in the intact heart: the concept of preload recruitable stroke work. Circulation 1985;71:994-1009.

24. Schiller NB, Shah PM, Crawford M, DeMaria A, Devereux R, Feigenbaum $\mathrm{H}$, et al. Recommendations for quantitation of the left ventricle by two dimensional echocardiography. J Am Soc Echocardiogr 1989;2:358-67.

25. Raff GL, Glantz SA. Volume loading slows left ventricular isovolumic relaxation rate. Circ Res 1981;48:813-24.

26. Suga H. Ventricular energetics. Physiol Rev 1990;70:247-77.

27. O'Brien PJ, Ianuzzo CD, Moe GW, Stopps TP, Armstrong PW. Rapid ventricular pacing of dogs to heart failure: biochemical and physiological studies. Can J Physiol Pharmacol 1990;68:34-9.

28. O'Brien PJ, Moe GW, Nowack LM, Grima EA, Armstrong PW. Sarcoplasmic reticulum Ca-release channel and ATP synthesis activities are early myocardial markers of heart failure produced by rapid ventricular pacing in dogs. Can J Physiol Pharmacol 1994;72:999-1006.

29. Cory CR, McCutcheon LJ, O'Grady M, Pang AW, Geiger JD, O'Brien PJ. Compensatory down-regulation of myocardial Ca channel in SR from dogs with heart failure. Am J Physiol 1993; 264:H926-37.

30. Spinale FG, Fulbright BM, Mukherjee R, Tanaka R, Hu J, Crawford FA, et al. Relationship between ventricular and myocyte function with tachycardia induced cardiomyopathy. Circ Res 1992;71:174-87.

31. Wolfe MR, Whitesell LF, Moss RL. Calcium sensitivity of isometric tension is increased in canine experimental heart failure. Circ Res 1995;76:781-9.

32. Weber KT, Pick R, Silver MA, Moe GW, Janicki JS, Zucker IH, et al. Fibrillar collagen and remodeling of dilated canine left ventricle. Circulation 1990;82:1387-401.

33. Weber KT, Anversa P, Armstrong PW, Brilla CG, Bumett JC Jr, Cruickshank JM, et al. Remodeling and reparation of the cardiovascular system. J Am Coll Cardiol 1992:20:3-16. 